\title{
The Study of Hematological Parameters of Animals in the Application of Enterosorbent Food Fiber
}

\author{
G. Tussupbekova ${ }^{1}$, Z. Yessimsiitova ${ }^{1}$, N. Ablaikhanova ${ }^{1}$, A. Rakhmetova ${ }^{2}$, G. Alshynbekova $^{2}$, \\ S. Tuleukhanov ${ }^{1}$, Z. Zhussupbekova ${ }^{2}$, G. Ashimhanova ${ }^{2}$ and Y. Kuandykov ${ }^{3}$ \\ ${ }^{1}$ Al-Farabi Kazakh National University, Almaty, Kazakhstan \\ ${ }^{2}$ Karaganda State University of the Name of Academician E.A. Buketov, Karaganda, Kazakhstan \\ ${ }^{3}$ Scientific and Practical Center of Sanitary and Epidemiological Expertise and Monitoring, Almaty, \\ Kazakhstan
}

\begin{abstract}
One of the most important issues is the study of healthy nutrition of the population of Kazakhstan. Proper nutrition provides growth, contributes to the prevention of diseases, increase efficiency and prolong life of people, while creating conditions for adequate adaptation to the environment. A large part of the population of Kazakhstan as a result of technological processing, the use of inferior in chemical composition of food raw materials, the influence of other causes, does not receive the necessary amount of essential components of food, which lead to illness, premature old age and shortened life. The situation is aggravated by the low cultural level of the population in terms of good nutrition and the lack of skills to lead a healthy lifestyle. The problem of quality and food safety, while food must have not only nutritional and physiological value, but also perform preventive functions.
\end{abstract}

Keywords: Hematological parameters, entersorbent, food, fiber, research.

\section{INTRODUCTION}

The deterioration of the environmental situation in many countries is accompanied by widespread pollution of the environment and food. The high level of contamination with toxic elements leads to the need to search for, develop, and introduce into food production prophylactic purposes aimed at removing toxic elements from the human body. From this point of view, it seems expedient to expand the range of products enriched with dietary fibers, in particular, entersorbents, which are able to excrete heavy and radioactive metals from the body. Considering that bread has an extremely important place in human nutrition, great attention is paid to the enrichment of bread with functional additives that give it therapeutic and prophylactic properties [1].

Bakery products in Kazakhstan are affordable, traditional, everyday food, improving their quality and nutritional value, the development of preventive products, functional and enriched, contribute to the implementation of the modern concept of healthy eating and expanding the range of products of the domestic food industry as a whole. The most appropriate and effective from a physiological, technological and economic point of view, the method

Address correspondence to this article at the Kazakh State National University of al-Farabi, Almaty, Kazakhstan; Tel: +77073851878;

E-mail: zhanat_2006@mail.ru of expanding the range of bakery products, increasing vitamin and mineral value, is the enrichment of their plant ingredients, which are a natural, balanced source of vitamins, dietary fiber, macro-and microelements

As enriching herbal ingredients, the use of local raw materials with accessibility, environmental cleanliness and wide distribution in the region is promising. From this point of view, rice husks containing vitamins, macro- and microelements, dietary fiber, organic acids and other substances deserve attention [2, 3].

Improving the recipe of bakery products using regional herbal ingredients is of great theoretical and practical interest and creates prerequisites for expanding the range, improving the quality, nutritional and biological value of the finished product $[4,5,6]$.

Considering this, the creation of bakery products enriched with biologically active substances, such as vitamins, dietary fiber, mineral elements and highgrade proteins of plant origin will significantly eliminate the deficiency of these ingredients in the human diet. In this regard, the development of the formulation and evaluation of consumer properties of a bakery product of functional purpose, enriched with vegetable dietary supplements, is relevant.

Ideal enterosorbent should be non-toxic, nontraumatic for mucous membranes, with good evacuation from the intestines, with high sorption

() 2019 SET Publisher 
capacity in relation to the removed components of chyme, so that the use of enterosorbents leads to minimal loss of beneficial ingredients.

Purpose of the study: to study the hematological parameters of animals with lead poisoning against the background of the use of enterosorbers food fibers.

\section{MATERIALS AND METHODS}

\section{The Selection Criteria of Animals}

Studies were carried out on white outbred rats, males weighing 180-230 $\mathrm{g}$, kept in normal vivarium conditions, 10 animals per cage with free access to food and water.

Laboratory methods, the purpose of which is to determine the nature of the biological action of the test substance, are performed on the experimental and control groups of animals. The animals stayed in quarantine for 10 days after delivery. Sick animals were not identified. No physiological abnormalities were observed, the coat was flat, pupils were not enlarged. Weight without any changes.

Steps of Administration of Food and Doses of Ingredients Monitoring of Progress of Health of Animals

The experiments were carried out in three stages:

- $\quad$ the first stage, the effect of Enterosorbers food fibers (EFF) (rice husk $(\mathrm{RH})$, carbonized rice husk $(\mathrm{CRH})$, crushed carbonized rice husk $(\mathrm{CCRH})$ on the functional state of the animal organism was determined, the biochemical and hematological parameters of blood were studied when EFF was used;

- the second stage of the experiment, the biochemical, morphological and hematological parameters of blood were studied using EFF (rice husk, carbonized rice husk, crushed carbonized rice husk) using a toxic poisoning model.

- the third stage, experimental evaluation of bakery products with EFF (rice husk, carbonized rice husk, crushed carbonized rice husk) was performed on the model of toxic poisoning.

Based on the lead acetate toxicity parameters, intragastrically $45 \mathrm{mg} \mathrm{/} \mathrm{kg}$ of lead acetate $\mathrm{Pb}$ $(\mathrm{OCOCH} 3)$ 2, EPV (rice husks $5 \mathrm{~g}$, carbonized rice husks $0.5 \mathrm{~g}$, carbonized ground rice was administered intragastrically to $1 \mathrm{ml}$ of groups II, III, IV, V in $1 \mathrm{ml}$ of distilled water Husk $0.5 \mathrm{~g}$ ) for 30 and 60 days.

During the experiment, the general condition of the rats was noted (body weight dynamics, rectal temperature). General condition was assessed by daily inspection of animals. Weighing, measuring rectal temperature, water and feed consumption was performed once a week.

The blood of experimental animals was collected in a vacutainer with K3EDTA, mixed 10 times to eliminate the formation of microbunches, and delivered to the laboratory. For the assessment of hematological parameters, the Complete blood count was used - a complete blood count on an automated hematology analyzer Abacus Junior 30 (Austria).Blood biochemical parameters were determined on a BioChem SA biochemical analyzer. The activity of enzymes was determined - the activity of amylase and lactate dehydrogenase, the content of total protein and glucose in blood serum.

\section{RESULTS AND DISCUSSION}

Dynamics of hematological indices of control and experimental animals with lead in them against the background of the use of EFF. To study the EFF, hematological blood parameters of experimental animals were analyzed. The results obtained during the experiment indicate a change in the hematological parameters of rats with lead acetate poisoning against the background of the use of EFF separately and in complex. During the experiment, the content of erythrocytes changed in the blood of experimental rats (Table 1).

As can be seen from the results of table 17 , this indicator had different values in each group. At the first stage in the third and fourth experimental groups, the content of erythrocytes by 30 days of the experiment increased by $3 \%$, and on the 60 th day of the experiment was higher than the control by 31 and $48 \%$, respectively (at $P<0,001$ ). At the second stage of the experiment, by the 30th day of the experiment, the content of erythrocytes in the blood in the second experimental group decreased by $22 \%$, for 60 days from the start of the experiment by 55\% ( $P<0,001)$ compared to the control group. The largest increase in this indicator was observed at the third stage of the experiment. On the 30th day of the experiment, the content of erythrocytes increased in the blood of rats in the third group by $14 \%$, in the fourth by $34 \%$ and in the 
Table 1: Dynamics of Red Blood Cells in the Blood of Rats, $10^{12} / \mathrm{L}(n=15)$

\begin{tabular}{|c|c|c|c|c|c|c|}
\hline \multirow[b]{2}{*}{ Timing } & \multicolumn{6}{|c|}{ Group of animals } \\
\hline & 1 & 2 & 3 & 4 & 5 & 6 \\
\hline \multicolumn{7}{|c|}{ The first stage of the experiment } \\
\hline 30 days & $6,10 \pm 0,03$ & $6,05 \pm 0,04$ & $6,33 \pm 0,07$ & $6,45 \pm 0,05^{*}$ & - & - \\
\hline 60 days & $5,71 \pm 0,02$ & $6,20 \pm 0,02$ & $7,50 \pm 0,02^{*}$ & $8,57 \pm 0,02^{*}$ & - & - \\
\hline \multicolumn{7}{|c|}{ The second stage of the experiment } \\
\hline 30 days & $6,02 \pm 0,01$ & $4,91 \pm 0,02^{*}$ & $5,82 \pm 0,05$ & $6,03 \pm 0,02$ & $6,27 \pm 0,02^{*}$ & - \\
\hline 60 days & $6,23 \pm 0,03$ & $4,01 \pm 0,01^{*}$ & $6,10 \pm 0,07$ & $6,57 \pm 0,06$ & $7,58 \pm 0,06^{*}$ & - \\
\hline \multicolumn{7}{|c|}{ The third stage of the experiment } \\
\hline 30 days & $6,12 \pm 0,02$ & $5,78 \pm 0,05^{*}$ & $6,97 \pm 0,03$ & $8,18 \pm 0,03^{*}$ & $7,62 \pm 0,06^{*}$ & $8,18 \pm 0,05^{\star}$ \\
\hline 60 days & $6,57 \pm 0,03$ & $6,00 \pm 0,03^{*}$ & $7,58 \pm 0,05^{*}$ & $9,94 \pm 0,05^{*}$ & $9,51 \pm 0,02^{*}$ & $9,47 \pm 0,03^{*}$ \\
\hline
\end{tabular}

(Note: statistically significant with respect to control $\left.{ }^{*} P<0,001\right)$.

fifth experimental group by $25 \%$ respectively ( $P$ $<0,001)$, and on day 60 in the third group of the experimental group the red blood cells was more by $15 \%$, in the fourth and fifth experimental groups by $51 \%$ and $45 \%$, respectively (at $P<0,001$ ). The increase in the number of red blood cells is accompanied by an increase in the concentration of hemoglobin (Figure 1). The lowest value of the index was observed on the 30th day of the experiment.

On the 60th day, this indicator increased in all groups, but its greatest value was recorded in the fourth experimental group. At the second stage, by the 30th day of the experimental study, the hemoglobin concentration in the fourth group increased by $9,3 \%$ compared with the control level. In the third experimental group, it increased by $12 \%$, respectively (at $P<0,001$ ). A similar increase in this indicator was recorded in the fifth experimental group. The highest hemoglobin concentration values were recorded by the 60th day of the experiment, the highest result was observed at the third stage in the 5th and 6th experimental groups. It exceeded the values of this indicator in other groups of experimental rats at different stages of the experiment.

When changing the content of erythrocytes in the blood of experimental rats, a decrease in the number of leukocytes was observed (Table 2). In the case of the removal of the blood with a lead and the use of EFF, the highest concentration of white blood bodies was recorded on the 30th day of the experiment. The concentration of leukocytes was the same for all experimental groups. Then their number began to decline and reached a minimum of 60 days. On the 30th day of the experiment at the first stage of the experiment, an increase in the number of leukocytes was recorded in rats of the second experimental group. It was $10 \%$ higher than the values for the control group and exceeded the values compared with the third

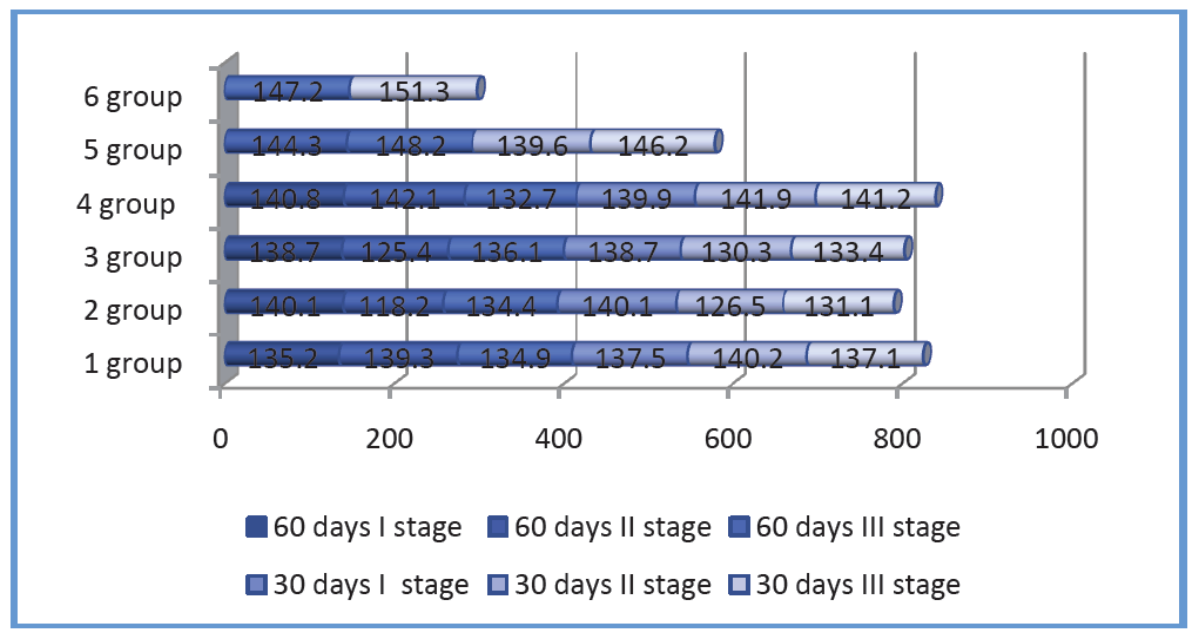

Figure 1: Dynamics of hemoglobin concentration in the blood of rats, $g / I(n=15)$. 
Table 2: Dynamics of the Content of Leukocytes in the Blood of Rats, $10^{9} / L(n=15)$

\begin{tabular}{|c|c|c|c|c|c|c|}
\hline \multirow{2}{*}{ Timing } & \multicolumn{6}{|c|}{ Group of animals } \\
\hline & 1 & 2 & 3 & 4 & 5 & 6 \\
\hline \multicolumn{7}{|c|}{ The first stage of the experiment } \\
\hline 30 days & $8,41 \pm 0,04$ & $9,28 \pm 0,03$ & $7,97 \pm 0,06$ & $9,32 \pm 0,05^{*}$ & - & - \\
\hline 60 days & $8,00 \pm 0,05$ & $10,97 \pm 0,04^{*}$ & $8,61 \pm 0,02$ & $9,65 \pm 0,05^{*}$ & - & - \\
\hline \multicolumn{7}{|c|}{ The second stage of the experiment } \\
\hline 30 days & $7,01 \pm 0,03$ & $5,78 \pm 0,04^{*}$ & $6,43 \pm 0,05$ & $6,20 \pm 0,01^{*}$ & $6,69 \pm 0,03^{*}$ & - \\
\hline 60 days & $8,30 \pm 0,01$ & $4,28 \pm 0,02^{*}$ & $5,11 \pm 0,02^{*}$ & $5,13 \pm 0,03^{*}$ & $6,29 \pm 0,04^{*}$ & - \\
\hline \multicolumn{7}{|c|}{ The third stage of the experiment } \\
\hline 30 days & $8,11 \pm 0,02$ & $9,01 \pm 0,03^{*}$ & $8,67 \pm 0,06$ & $9,67 \pm 0,06$ & $10,67 \pm 0,03^{*}$ & $11,47 \pm 0,03^{*}$ \\
\hline 60 days & $9,20 \pm 0,03$ & $11,30 \pm 0,01^{*}$ & $7,23 \pm 0,07^{*}$ & $9,30 \pm 0,07$ & $11,09 \pm 0,01^{*}$ & $12,0 \pm 0,01^{*}$ \\
\hline
\end{tabular}

(Note: statistically significant with respect to control * $P<0,001$ ).

experimental group by $16 \%$, respectively (with $P$ $<0,001)$. On the 60th day of the experiment, this indicator was the highest in the second experimental group, its value increased by $37 \%$ and decreased to $10,97 \pm 0,04 \times 10^{9} / \mathrm{L}(\mathrm{P}<0,001)$ compared with the control group.

At the second stage of the experiment, the minimum value of leukocytes was recorded in the third experimental group by 30 days it was $21 \%$ lower than the value for the control group, on the 60th day of the experiment it was also 1,9 times less and was $4,28 \pm 0,02 \times 10^{9} / L(P<0,001)$ compared with the control group.

In the subsequent stages of the experiment, the maximum of this indicator was observed in the third experimental group. Its minimum value was fixed on the 60th day of the experiment and amounted to $7,23 \pm 0,07 \times 10^{9} / \mathrm{L}$, respectively (at $\mathrm{P}<0,001$ ). The maximum of this indicator was observed by 30 days of an experimental study, in the fourth by $19 \%$ $\left(9,67 \pm 0,06 \times 10^{9} / \mathrm{L}\right)$ and in the fifth experimental group by $32 \%\left(10,67 \pm 0,03 \times 10^{9} / L\right)$, respectively $(P<0,001)$. On the 60th day of the experimental study in the fourth experimental group compared with the control it was $\left(9,30 \pm 0,07 \times 10^{9} / \mathrm{L}\right)$ and the fifth experimental group statistically significantly increased from $10,67 \pm 0,03 \times 10^{9} / \mathrm{L}$ to $11,09 \pm 0,01 \times 10^{9} / \mathrm{L}$, respectively (at $\mathrm{P}<0,001)$.

The combined use of lead and EPV (rice husk, carbonized husk, crushed carbonized husk) contributes to platelet count in the blood of animals. In the course of the experiment, the value of this indicator increased in all experimental groups (Figure 2).

The minimum platelet count was observed on the thirtieth day of the experiment and the platelet count increased in almost all experimental groups. At the first

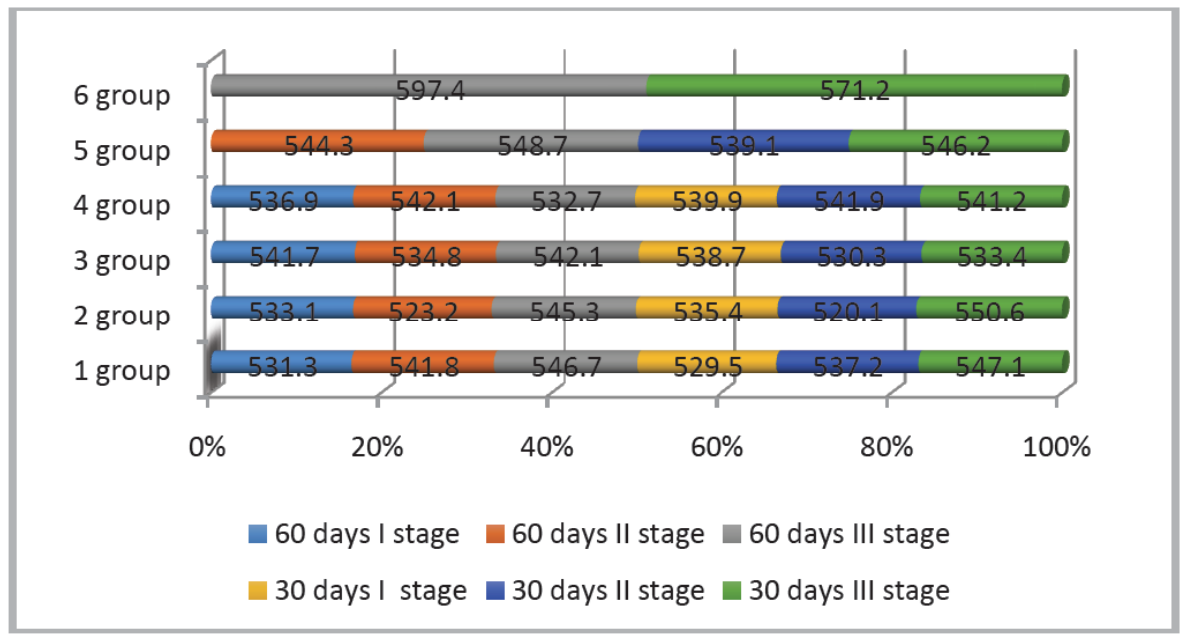

Figure 2: Dynamics of platelet content in the blood of rats, $109 / \mathrm{I}(\mathrm{n}=15)$. 
stage of the experiment in the second experimental group, this indicator increased by $3,1 \%(P<0,001)$ compared with the control group. In the third experimental group, the value of the indicator under review decreased by $1,5 \%$. In the fifth experimental group, the indicator increased by $10 \%$, respectively. By the 60th day of the experiment, the value of this indicator in the second experimental group increased compared with the control by $2,4 \%$, respectively (at $\mathrm{P}$ $<0,001)$.

At the second stage of the experimental study in the fifth experimental group, on the 30th day, its increase was $5,2 \%$, on the 60 th day of the experiment, the platelet count increased by $8,1 \%$ and amounted to $597,41 \pm 39,82 \times 10^{9} / \mathrm{L}$ compared to the control group. High platelet counts were observed in all experimental groups on the thirtieth day in the third stage of the experiment. The maximum concentration of these blood cells was recorded in the fifth experimental group. However, this fact, like the above data on platelet concentration, is not reliable.

When the content of blood cells in the body changes, the dynamics of its hematocrit are performed (Figure 3).

In the case of an increase in the concentration of erythrocytes and platelets, the total volume of protein aggregates increases and the ratio between this volume and total blood volume increases. But it may decrease the number of leukocytes. At the first stage of the experiment, the hematocrit was the same for all groups of experimental rats. On the 60th day of the experimental study, its value decreased in the third experimental group by $5 \%$, respectively (at $\mathrm{P}<0,001$ ). In the fourth experimental group, it decreased by $4,5 \%$.
In the fifth experimental group, the value of this indicator decreased by $1,5 \%$, respectively $(P<0,001)$.

At the second stage, on the 30th day of the experimental study in the second experimental group, the hematocrit decreased by $15 \%$ and amounted to $41, .09 \pm 0,74 \%(P<0.001)$ compared with the control group. At the same time, in group 3 and 4 , the indicator changed by 3,1 and $1,2 \%$, respectively.

At the final stage of the experiment, the hematocrit had the highest values in the third experimental group compared with the data obtained during some previous stages. In the third group, hematocrit decreased by $2,4 \%$ relative to the value for 30 days. In the fourth experimental group, the value of this indicator decreased by $15 \%$ and was $5,5 \%$ less than the value for 30 days. In the fifth experimental group, the hematocrit increased by $21 \%$ in relation to the result for 30 days, and it exceeded the value of the previous stage by $7 \%$.

Thus, the results obtained indicate that the use of the investigated enterosorbers food fibers (rice husk, carbonized husk, crushed carbonized husk) has a more favorable effect on the organism of rats.

Currently, the study of methods to improve the health and quality of life of the population of Kazakhstan, especially those living in areas of environmental ill-being and in contact with harmful factors, has exacerbated the problem of preserving human health and there is a need to develop formulations of new types of functional foods. Unfortunately, despite the abundance of experimental studies on the problem of creating bakery products for functional purposes, many of the issues of introducing

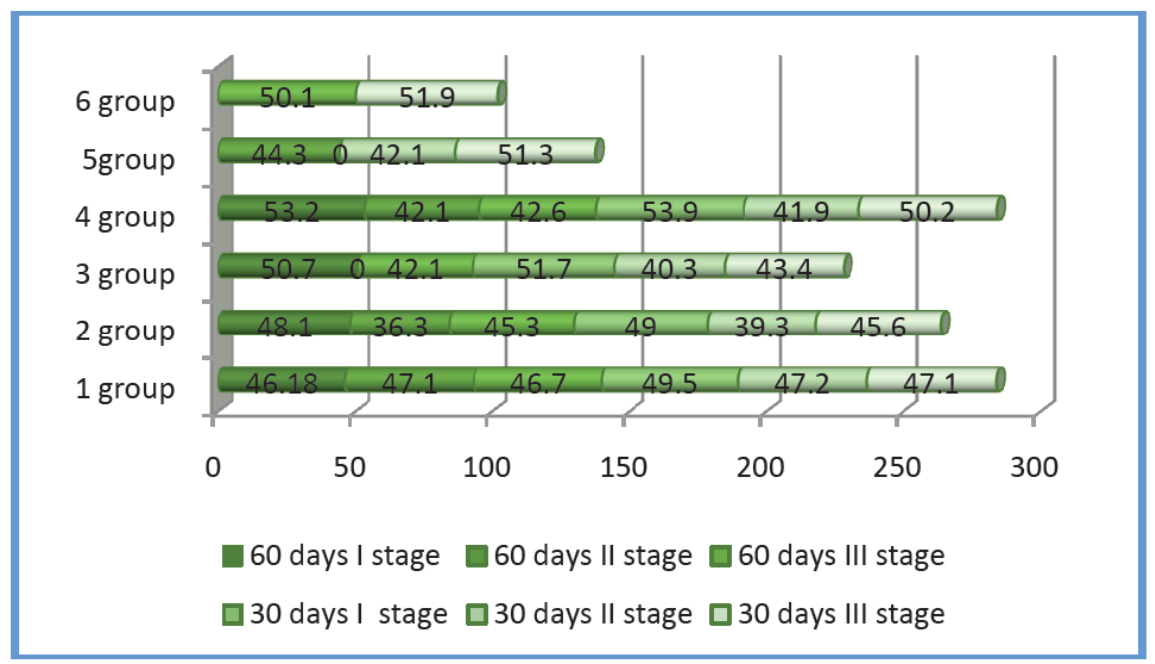

Figure 3: Dynamics of hematocrit of rats, $\%(n=15)$. 
into the recipe and the effects of the components introduced on the consumer properties of finished products remain insufficiently studied. In this regard, the development of formulations and assessment of consumer properties, functional bakery products, enriched with herbal dietary supplements, is relevant.

The study examined hematological research methods using prophylactic grain-based supplements with the addition of natural enterosorbing dietary fibers with desired multifunctional properties, with targeted detoxifying properties that reduce the risk of poisoning the body with heavy metal salts, radio radulants, pesticides and improve the quality of life of the population. The perspective and practical significance of the study shows the process of obtaining a wide range of new dietary fibers, which will be used to meet the needs of different categories of the population in effective prevention and treatment of poisoning, allergies, intestinal infections, skin diseases, intoxication of various origins.

As a result of observation in the experiment of animals hounded with lead acetate at a dose of $45 \mathrm{mg} /$ $\mathrm{kg}$, the physiological state of white rats was characterized by depression, decrease in motor activity, fearfulness, overcrowding. In the middle of the month, conjunctivitis and rhinitis were accompanied by the release of catarrhal exudate. By the end of the month of the experiment, a decrease in body weight by an average of $10 \%$ was noted, as well as ruffled hair, and a progressive decrease in body weight and feed consumption was observed. On the 15th day of the experiment, the clinical picture of lead rat poisoning in white rats was accompanied by a decrease in activity, the pallor of the visible mucous membranes, the urine acquired an orange-brown color, and in some animals there was a dramatic depletion and refusal of food. On the 15th day of the experiment, these symptoms of intoxication increased, in some rats exhaustion, baldness, and aggressiveness were observed. On the 60 th day of the experiment, partial recovery of motor activity and food excitability was observed in the surviving experimental animals. A loss was not observed.

Research results substantiate the effect of EFF on the morphological parameters of blood. The actions of these drugs contribute to changes in the content of platelets in the endogenous environment of the macroorganism. There is an increase in the number of red blood cells, contributing to the dynamics of physiological processes. The intensity of gas exchange and the transport of substances dissolved in the blood plasma increases. As a result, the activity of biochemical transformations based on the decomposition and synthesis of nutrients increases. The formation of substances of complex composition improves the processes of cell division, since they constitute the membrane membrane. Also, this drug form the structure of organoids. Therefore, an increase in the number of macromolecules, as well as molecules that are simpler in their structure, increases the activity of various processes that ensure the constancy of the chemical composition of the internal environment of the organism. This increases the antagonism of exogenous factors due to the influence of various pathogenic processes. This fact determines the possibility of optimizing the number of leukocytes and platelets, which form the basis of the humoral factor and the constancy of the internal environment with active changes in exogenous parameters [29].

According to the research results, an active increase in the number of red blood cells was observed in the composition of the dispersed phase of blood. The most intensively considered indicator increased with the introduction of EPO into the diet of rats. Entering the digestive tract, the EPO were absorbed by the intestinal villi and entered the endogenous medium of the microorganism. After binding to the composition of the biologically active substance, EPO participated in the regulation of erythropoiesis, contributing to the activation of the formation of erythrocytes. According to the data obtained with the use of EPO, the average volume of erythrocytes increased, as well as the amount of hemoglobin in the erythrocyte. However, against the background of an increase in the content of erythrocytes, changes in concentrations of leukocytes and platelets are possible, which will affect the parameters of blood functionality.

The results of our own research confirm the dynamics of the content of other blood cells. In the course of the experiment, a decrease in the concentration of leukocytes in the blood of rats was carried out at the maximum, together with the joint use of lead acetate and EFF at 30 days. Such dynamics may be due to the effect of lead acetate on leukopoiesis. As the experimental rats grew in their bodies, the number of mature segmented neutrophils increased, capable of neutralizing potential pathogens of various infectious diseases. The total number of leukocytes in the rats decreased. This fact may be due to the activation of the action of lead acetate and EFF on the maturation process of white blood platelets. The concentration of platelets in the blood of rats actively increased over the course of the experiment. The use 
of the studied drugs contributed to the most intensive growth of the factor that determines blood clotting. In terms of its own volume, platelets are significantly smaller than leukocytes and red blood cells. Also, their number is a thousand times smaller than the number of red blood cells.

However, they affect the structural and functional potential of the dispersed blood phase, regulating the level of constancy of the entire endogenous environment. At the same time, platelets perform the compensatory function of immune activity. The increase in the number of platelets due to the intensifying effect of EFF on thrombopoiesis. An increase in the content of erythrocytes and platelets in the blood of experimental animals significantly increases the functionality of the liquid connective tissue, but this increases the viscosity of the dispersed system. This increases the load on the work of the cardiovascular system, and also reduces the oxygen transport of bio-organic molecules to various anatomical parts of the body. Therefore, it is necessary to increase the amount of water in the composition of the blood dispersion medium. When using EFF, the intoxication of cells of the host organism is significantly reduced, which contributes to the additive distribution of moisture between the components of the endogenous environment. This increases the amount of the liquid phase of the blood. However, the volume of the solid phase also increases. As a result, an increase in hematocrit occurs $[7,8]$.

Studies of the biological activity of joint use of rice husk + crushed carbonized rice husk confirm the growth of rat hematocrit. These substances have an activating effect on hemopoiesis due to the intensification of the processes of formation and development of blood cells. This is confirmed by comparing the experimental data for the control and experimental groups. However, if we consider the age dynamics of the indicator in the control group, then there is an increase in hematocrit at the end of the experiment compared with the result for the penultimate time stage. This may be due to a decrease in the amount of water in the composition of the blood plasma against the background of an increase in the amount of substance of the dispersed phase. The recorded process is due to the activity of the harmful microflora of the digestive tract, contributing to the dehydration of the internal environment of the microorganism.
The growth of the dispersed phase is due to an intensive increase in the number of leukocytes. At the same time, in the second stage of the experiment, uniform dynamics of the blood composition of the experimental rats is carried out. As a result, improvement is possible, as the physiological status of the organism.

\section{CONCLUSION}

- It has been established that the use of EFF has a significant effect on the hematological parameters of the blood of animals and an increase in the content of red blood cells, platelets, hemoglobin in the blood has been detected, and the hematocrit in it is at a higher level, which objectively reflects the level of metabolic processes and the functional state of the body of rats;

- It was established that EFF in combined use of rice husk composite with carbonized rice husk, crushed carbonized rice husk has high adsorption activity, antioxidant, anti-toxic and therapeutic-prophylactic properties, this was especially manifested when used with crushed carbonized rice husk.

- $\quad$ It is effective and useful.

\section{REFERENCES}

[1] Sanina T. Increasing the nutritional value of bakery products of mass consumption. Bakery of Russia 2006; 6: 28-29.

[2] Alekseenko E. Unconventional natural raw materials for the production of bakery products. Bakery Products 2008; 9: 5051.

[3] Dudkin MS, Schelkunov LF. Dietary Fiber and New Foods. Food Supply Ques 1998; 2: 35-41.

[4] Pogozheva AV. Dietary fibers in preventive medical nutrition. Food Supply Ques 1998; 1: 39-42.

[5] Tutelyan VA. Biologically active food supplements: past, present, future. Nutrition and health. Biologically active food supplements: Proc. report II International Symposium. M., 1996; 164-166.

[6] Berezhnova, Larisa V. Hygienic aspects of using bread with improved sorption properties in the nutrition of the population: Author. Cand. Med. sciences. - Moscow 2005; p. 22.

[7] Bazeliuk LT, Amanbekova AU, Tusupbekova GA. Cytochemical parameters in studying the body at peripheral hemopoiesis level. Meditsina truda i promyshlennaia ekologiia 2005; 4: 5-12.

[8] Pletneva TM, Potapova NI. Heavy metals and standardization of infusions. Pharmacia 2014; 4: 9-10. 